\title{
Intervención psicológica en personas con enfermedad renal crónica en hemodiálisis
}

\author{
Psychological intervention in people \\ with chronic kidney disease in hemodialysis
}

\author{
Mariana Becerril González ${ }^{1}$ \\ Autor para correspondencia: Mariana Becerril González, marii.becerril@hotmail.com.
}

\begin{abstract}
RESUMEN
Introducción: La enfermedad renal crónica es un padecimiento que deteriora el funcionamiento de los riñones de manera constante, progresiva e irreversible; por ello, es necesario fomentar la adherencia al tratamiento a favor de la calidad de vida y mejorar así los mecanismos de adaptación de quienes lo sufren. Objetivo: Incrementar la adherencia al tratamiento y los mecanismos de adaptación psicológicos, afectivos y sociales en adultos con enfermedad renal crónica en etapa 4-5 dentro del programa de hemodiálisis de un hospital de alta especialidad del Estado de México. Método: Se evaluó a un total de 16 personas con la Escala de Adherencia Terapéutica, y los mecanismos de adaptación de las personas con ERC en terapia de hemodiálisis, aplicándoseles un programa de once sesiones grupales por semana, con enfoque cognitivo-conductual y terapia racional emotiva. Resultados: Las puntuaciones de preevaluación y postevaluación mostraron que los mecanismos de adaptación aumentaron significativamente, y asimismo el puntaje de adherencia al tratamiento, manteniéndose el control de ingesta de medicamentos y alimentos. Discusión: El programa es útil en la muestra para incrementar los mecanismos de adaptación y adherencia al tratamiento.
\end{abstract}

Palabras clave: Enfermedad renal crónica; Adherencia al tratamiento; Mecanismos de adaptación.

\begin{abstract}
Introduction: Chronic kidney disease (CKD) is a disease that seriously compromises functioning of the kidneys in a constant, progressive, and irreversible way. Thus it is necessary to increase adherence to medical treatments in order to improve quality of life and adaptation mechanisms of such patients. Objective: To increase treatment adherence and psychological, affective, and social adaptation mechanisms in adults with chronic kidney disease in stage 4-5 on hemodialysis. Method: Through a quasi-experimental design, the effects of an intervention were evaluated on the Therapeutic Adherence Scale and the adaptation mechanisms on 16 patients with CKD in hemodialysis. A program of 11 weekly group sessions with cognitive-behavioral and rational-emotive therapy was applied. Results: Pre and post-evaluation scores showed that coping mechanisms increased significantly; treatment adherence score increased and medication and food intake control was maintained. Discussion: The program was useful to increase coping mechanisms and adherence to treatment.
\end{abstract}

Keywords: Chronic kidney disease; Adherence to treatment; Adaptation mechanisms.

\footnotetext{
${ }^{1}$ Retorno Bosques de Brasil 18, Bosques de la Hacienda, 2 Sección, Cuautitlán Izcalli, Edo. de México, México, tel. (55)55-44-70-41-64, correo electrónico: marii.becerril@hotmail.com y marianabecerrilg10@gmail.com.
} 
Recibido: $22 / 04 / 2020$

Aceptado: 20/06/2020

$\int^{1}$ 1 ser humano es un ente biopsicosocial que cuando se ve afectada la salud, ve también perjudicado su desarrollo óptimo; asimismo, cuando experimenta alteraciones en su salud mental, entendida como la capacidad de afrontar las tensiones de la vida cotidiana y de ser funcional en su contexto social, experimenta también una alteración en su bienestar individual (Organización Mundial de la Salud [OMS], 2016).

La enfermedad renal crónica (ERC en lo sucesivo) deteriora gradual y progresivamente el funcionamiento de los riñones al reducirse el número de nefronas que realizan las funciones excretoras, depurativas y metabólicas (Pascual et al., 2017). Se desarrolla como consecuencia de padecimientos renales primarios, glomerulonefritis, quistes urológicos, hipoplasia renal y nefropatía diabética, entre otros, por lo que es la segunda causa de muerte en México con más de 101 mil personas fallecidas el año 2018 (Instituto Nacional de Estadística y Geografía [INEGI], 2018). Las repercusiones impactan en distintas áreas de las personas con ERC; por ejemplo, padecen anemia, apatía, desubicación espacial, alucinaciones, irritabilidad, depresión, ansiedad. Los enfermos disminuyen sus actividades y se ven sometidos a restricciones dietéticas (Ortiz y Riobó, 2012).

La ERC se evalúa en una progresión de cinco estadios de deterioro de la función renal a través de los cuales se valora el filtrado glomerular estimado y albuminuria de la persona con ERC. En cada estadio se calculan ciertas características específicas, y es en el estadio 4-5 donde se considera y prepara un tratamiento de sustitución renal o un tratamiento paliativo (Martínez et al., 2014).

La enfermedad renal tiene alta morbilidad; de hecho, cerca de una décima parte de la población mundial se ve afectada por esta enfermedad. La Sociedad Latinoamericana de Nefrología e Hipertensión (SLANH) de América Latina reportó que 613 pacientes por cada millón de habitantes tuvieron un tratamiento para la sustitución de la función renal en el año 2011 (Organización Panamericana de la Salud, 2015). En México las cifras son alarmantes, ya que es una enfermedad que por más de cuatro años ha formado parte de las diez causas de mortalidad más importantes. En 2018 hubo al menos 13,875 defunciones a causa de insuficiencia renal (INEGI, 2018). Es por este motivo que resulta importante su estudio para conocer sus consecuencias afectivo-emocionales y brindar a los pacientes la atención psicológica requerida.

Ante este panorama desgastante para las personas con ERC se requieren intervenciones especializadas para mejorar o mantener la calidad de vida. Contar con un equipo multidisciplinario en el que cada trabajador de la salud aporte sus conocimientos y sume sus esfuerzos para brindar un tratamiento integral a las personas con ERC es uno de los puntos clave para un tratamiento exitoso (Molina, Sánchez, Castro y Morera, 2014).

El conocimiento aportado por diversas investigaciones subraya el papel de la psicología de la salud en la investigación y resolución de problemas relacionados con los aspectos subjetivos y comportamentales relacionados con el proceso de salud-enfermedad (Martín y Grau, 2014). Los psicólogos auxilian en el alivio de los malestares emocionales, conductuales y sociales, y en el caso de enfermedades degenerativas pone particular atención en la depresión y la ansiedad, frecuentes en las personas (García, Bados y Saldaña, 2012; OMS, 2017). Utilizan también los recursos de la tanatología y de los cuidados paliativos incidiendo en el sentido de la vida a partir de la enfermedad, utilizando para ello las potencialidades que se detectan en la persona con ERC (Ramírez, 2016).

El psicólogo de la salud trabaja sobre variables que influyen en la falta de adherencia al tratamiento, entendido este como el conjunto de comportamientos efectivos para cumplir con las prescripciones médicas (farmacológicas y no farmacológicas) que se requieren para el control de la enfermedad (Soria, Vega y Nava, 2009), debido a que la persona con ERC sufre alteraciones psicológicas de tipo cognitivo, afectivo-emocional y conductual que lo llevan a sabotear el tratamiento (García et al., 2016). De igual manera, para los psicólogos es necesario promover los mecanismos de adaptación a la enfermedad, entendida como la habilidad y capacidad de una persona para responder al cambio en su estilo de vida y para adaptarse 
a una situación estresante manteniendo su integridad orientada hacia la salud y el crecimiento (Devia, Falcón, Farfán y Vargas, 2010).

Diversas investigaciones sobre la intervención psicológica en personas con ERC demuestran sus beneficios. La OMS inició en 2004 esta iniciativa, creando para ello una guía de trabajo para padecimientos prolongados, la cual tiene como propósito fomentar la adherencia terapéutica a partir de intervenciones múltiples en pacientes, prestadores de servicio y el propio sistema de salud; Así, por ejemplo, a partir de la terapia racional emotiva Ecuador trabajó con distorsiones cognitivas que afectaban el estado de ánimo, comprobándose que disminuían los síntomas depresivos (Córdoba, 2010). En México se aplicó un programa psicoeducativo para mostrar cambios en el nivel de conocimientos sobre la insuficiencia renal crónica buscando un sentido positivo (Torres, 2010); Mendoza, González, Cabrera y Arzate (2016) dieron a conocer que existen variables asociadas a la calidad de vida en las que es necesario intervenir, como la depresión, la ansiedad y la soledad, con el fin de mejorar la actitud hacia el tratamiento y la adherencia, de modo que permitiese gozar de una buena salud mental. En España, específicamente en Islas Canarias, se llevó a cabo una intervención psicológica para enfermos crónicos a través del uso de refranes, cuyos resultados mostraron una mejor postura sobre la manera de vivir, entendiendo mejor la enfermedad de los participantes (Pelechano, 2012); mientras que en Barcelona se implementó un programa de actividades recreativas en hemodiálisis, mostrando que los programas lograron reducir el estrés, la ansiedad y la depresión reportados al inicio por los 37 participantes (Pedreira et al., 2020). En Sevilla también se hizo un estudio sobre el impacto del tratamiento psicoterapéutico a partir de la psicoterapia gestalt, cuyos resultados mostraron disminuciones en los índices de depresión de los pacientes en hemodiálisis, aumento en la adherencia al tratamiento y una relación entre los indicadores psicológicos y fisiológicos en las personas con ERC (Salas y Fernández, 2019).

Sin importar el enfoque desde el cual se efectúe la intervención, las personas generarán sus propios beneficios, y es por ese motivo que la presente investigación con enfoque cuantitativo, explicativo y diseño cuasiexperimental, tuvo como objetivo mejorar los conocimientos que actualmente se tienen sobre los programas de intervención grupales en personas con ERC en tratamiento de hemodiálisis con técnicas cognitivo-conductuales y terapia racional emotiva, a fin de acrecentar la adherencia al tratamiento y los mecanismos de adaptación psicológicos, afectivos y sociales en adultos con ERC en etapa 4-5 dentro del programa de hemodiálisis de un hospital de alta especialidad del Estado de México (México).

\section{MÉTODO}

\section{Participantes}

Participaron pacientes con ERC del programa de hemodiálisis del hospital de los turnos matutino y vespertino, quienes fueron invitados a participar, principalmente con ERC del segundo y tercer bloque, en un periodo de ocho meses. Al principio, 27 personas con ERC fueron reclutadas, aunque únicamente 16 concluyeron el programa de intervención psicológica.

Se excluyó a aquellos que no estuvieran en el programa de hemodiálisis, que no pudieron asistir o que no estuvieron interesados, así como a los participantes que faltaran a cuatro sesiones consecutivas.

\section{Instrumentos}

Escala de Adherencia Terapéutica (Soria, Vega y Nava, 2009).

Este instrumento consta de 21 ítems puntuados de 0 a 100; se divide en tres categorías: Control de ingesta de medicamentos y alimentos, Seguimiento médico conductual y Autoeficacia. Es una escala que, para calificarla, se suman el valor de las respuestas; tiene un coeficiente alfa de Cronbach de .91 y está adaptada a población mexicana.

\section{Mecanismos de Adaptación de los Pacientes con ERC en Terapia de Hemodiálisis}

(Devia, Falcón, Farfán y Vargas, 2010).

Este cuestionario evalúa los mecanismos psicológicos, afectivos y sociales. Estandarizado para po- 
blación mexicana, tiene un coeficiente alfa de 0.7 y consta de 33 enunciados que se valoran en una escala tipo Likert.

\section{Análisis estadístico}

Se capturaron las respuestas de los instrumentos en el programa SPSS para llevar a cabo un análisis de las tres categorías de la Escala de Adherencia Terapéutica; para el análisis total de adherencia, se evaluó el puntaje del nivel como baja, moderada o alta, efectuándose el cálculo del nivel comprometido, compensatorio e integrado en los mecanismos de adaptación. En cada análisis se obtuvieron medidas de tendencia central. Asimismo, se efectuó un análisis para muestras relacionadas con el fin de comparar los resultados de la preevaluación y la postevaluación; se realizó también una correlación entre los citados instrumentos.

\section{Procedimiento}

Este trabajo se llevó a cabo en el área de hemodiálisis del hospital, en el que la población total de personas con ERC fue de 98.

Se realizaron once sesiones semanales a pie de cama durante la primera hora del tratamiento, y otras antes de que los participantes ingresaran a hemodiálisis, todas ellas de forma grupal. El programa de intervención tuvo un enfoque cognitivo-conductual y de terapia racional emotiva, por lo que, utilizando las técnicas de modificación de conducta, se abordan de igual manera la parte emocional de las reacciones de los individuos para aumentar la adherencia al tratamiento y los mecanismos de adaptación psicológicos, afectivos y sociales.

Este estudio cumplió en todo momento con el código de ética y estuvo apegado a las consideraciones éticas y lineamientos de investigación en el área médica. Se explicó previamente a todos los participantes los objetivos y forma de trabajar, al final de lo cual todos ellos firmaron un consentimiento informado del trabajo psicológico en el que participarían.

\section{RESULTADOS}

Siguiendo los objetivos del estudio y los criterios de participación, se incluyeron 16 participantes. La Tabla 1 muestra los datos sociodemográficos de la población analizada.

Tabla 1. Datos sociodemográficos de los participantes.

\begin{tabular}{|c|c|c|}
\hline \multirow{2}{*}{ Sexo } & Masculino & $69 \%$ \\
\hline & Femenino & $31 \%$ \\
\hline Edad & \multicolumn{2}{|l|}{$\begin{array}{l}+36.7 \\
\sigma=15\end{array}$} \\
\hline \multirow{4}{*}{$\begin{array}{l}\text { Tiempo desde } \\
\text { el inicio de la ERC }\end{array}$} & 1 a 2 años & $37.5 \%$ \\
\hline & 3 a 4 años & $43.8 \%$ \\
\hline & 5 a 6 años & $12.5 \%$ \\
\hline & 8 años y más & $6.3 \%$ \\
\hline \multirow{4}{*}{ Ocupación } & Trabaja & $63 \%$ \\
\hline & Hogar & $25 \%$ \\
\hline & Pensionados & $6 \%$ \\
\hline & Estudiantes & $6 \%$ \\
\hline \multirow{2}{*}{ Estado civil } & Soltero(a) & $50 \%$ \\
\hline & Casado(a) & $50 \%$ \\
\hline \multirow{4}{*}{ Grado académico } & Secundaria & $44 \%$ \\
\hline & Primaria & $31 \%$ \\
\hline & Nivel superior & $19 \%$ \\
\hline & Medio superior & $6 \%$ \\
\hline
\end{tabular}

En la Gráfica 1 se presentan los resultados comparativos de la adherencia al tratamiento. Se obtuvo un aumento en el promedio total de $4.7 \%$ y en sus categorías: control de ingesta de medicamentos y alimentos, 2.7\%; seguimiento médico, 1.3\%, y autoeficacia, $10.5 \%$.

En la Gráfica 2 se muestra una comparación de los resultados obtenidos respecto a los mecanismos de adaptación; al final hubo un incremento del promedio inicial de $9.1 \%$; la subcategoría de mecanismos psicológicos aumentó en $3.7 \%$ en la postevaluación, la de mecanismos afectivos en $1 \%$ y la de mecanismos sociales en $3.7 \%$.

Para evaluar la relación entre variables, se efectuó una correlación bivariada entre el EAT y el referente a los mecanismos de adaptación, de la preevaluación a la postevaluación, para comprobar la existencia de una estrecha relación entre ambas variables, cuyas correlaciones se muestran en la Tabla 2. 
Gráfica 1. Comparación preevaluación y postevaluación de adherencia al tratamiento en personas con ERC en hemodiálisis.

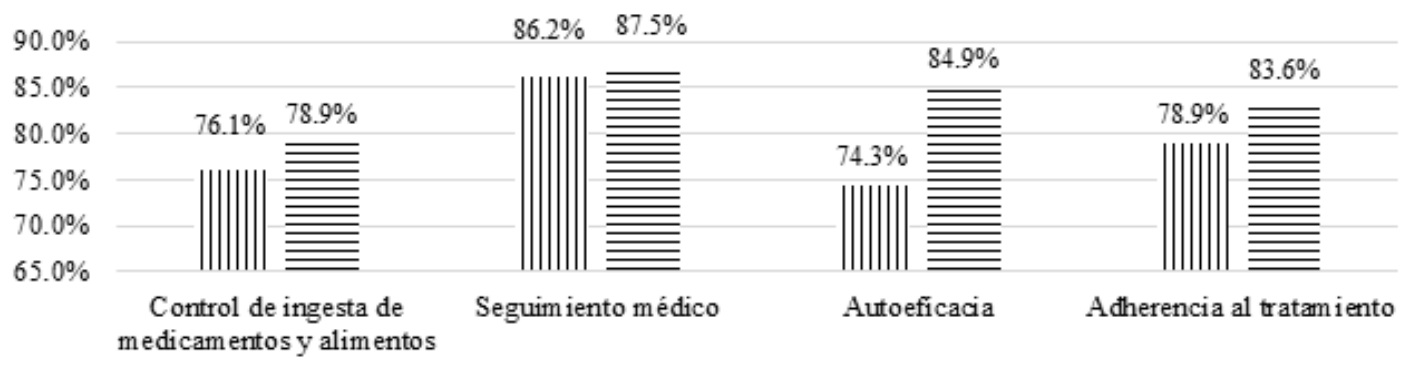

I Preevaluación = Postevaluación

Gráfica 2. Comparación de preevaluación y postevaluación de los mecanismos de adaptación en personas con ERC en hemodiálisis.

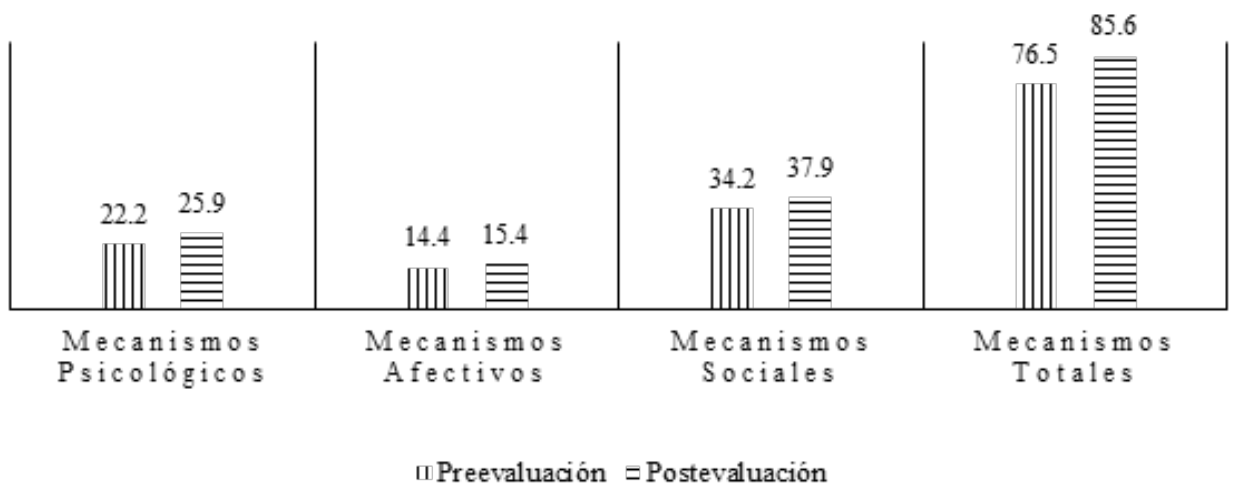

Tabla 2. Correlaciones de la preevaluación y postevaluación de las personas con ERC en hemodiálisis.

\begin{tabular}{|c|l|c|c|c|c|}
\cline { 3 - 6 } \multicolumn{2}{c|}{} & $\begin{array}{c}\text { Adherencia } \\
\text { total }\end{array}$ & $\begin{array}{c}\text { Control de ingesta } \\
\text { de medicamentos } \\
\text { y alimentos }\end{array}$ & $\begin{array}{c}\text { Seguimiento } \\
\text { médico }\end{array}$ & Autoeficacia \\
\hline \multirow{4}{*}{ Preevaluación } & Mecanismos & $.63^{* *}$ & .35 & .06 & $.67^{* *}$ \\
\cline { 2 - 6 } & Psicológicos & $.61^{*}$ & .35 & .04 & $.70^{* *}$ \\
\cline { 2 - 6 } & Afectivos & $.54^{*}$ & .41 & .13 & .47 \\
\cline { 2 - 6 } & Sociales & .33 & .09 & .02 & .45 \\
\hline \multirow{4}{*}{ Postevaluación } & Mecanismos & $.70^{* *}$ & $.63^{* *}$ & $.67^{* *}$ & .40 \\
\cline { 2 - 6 } & Psicológicos & .38 & .47 & .21 & .19 \\
\cline { 2 - 6 } & Afectivos & .30 & $.50^{*}$ & .02 & .19 \\
\cline { 2 - 6 } & Sociales & $.58^{*}$ & .32 & $.75^{* *}$ & .36 \\
\hline \multirow{2}{*}{$* * * p \leq 0.001 ; * * p \leq 0.01 ; * p \leq 0.05$} & & & & \\
\hline
\end{tabular}

En la preevaluación, hubo una correlación significativa entre los mecanismos de adaptación con la adherencia al tratamiento y la autoeficacia; las subcategorías del instrumento también obtuvieron una correlación significativa entre los mecanismos psicológicos y la adherencia al tratamiento y la autoeficacia, y mecanismos afectivos con adhe- rencia al tratamiento. Tras la aplicación del programa de intervención psicológica, las correlaciones significativas fueron entre los mecanismos de adaptación y la adherencia al tratamiento, el control de ingesta de medicamentos y alimentos, y el seguimiento médico; las subcategorías de mecanismos afectivos correlacionaron con el control 
de ingesta de medicamentos y alimentos, y los mecanismos sociales con la adherencia total y el seguimiento médico.

\section{DISCUSIÓN}

Con base en el objetivo del estudio, que fue aumentar la adherencia al tratamiento y los mecanismos de adaptación psicológicos, afectivos y sociales en adultos con ERC en etapa 4-5 en un programa de hemodiálisis, es posible apreciar que el programa de intervención de once sesiones ejecutado grupalmente demostró su funcionalidad en los 16 participantes, pacientes de un hospital de alta especialidad del Estado de México. Los puntajes obtenidos así lo demuestran, como en otros programas que han obtenido resultados similares.

En la presente investigación algunas de las temáticas que mostraron éxito fueron, adherencia a la dieta, registros conductuales de hábitos alimenticios, conocimiento sobre la enfermedad, diario de emociones, técnicas de respiración, técnicas de autocontrol; y algunas de esta en el estudio de Rodríguez, Rentería y García (2013), también permitieron obtener respuestas favorables por parte de las personas en la adherencia a la dieta.

En cuanto a la adherencia al tratamiento, tras la aplicación del programa de once sesiones las personas con ERC se vieron más comprometidas con su estado de salud, por lo que acudieron de manera regular a las sesiones de hemodiálisis, consultas médicas, toma de medicamentos y control de la alimentación; también aumentó su autoeficacia, mostrándose más seguros de sí mismos, de su capacidad de realizar actividades y de responsabilizarse de su propio proceso salud-enfermedad.

Es importante señalar que según Oblitas (2009) y García et al. (2016), las funciones del psicólogo deben incluir también las de la psicoeducación sobre la enfermedad para generar cambios, recomendación que se puso en práctica en el programa porque resulta esencial para que el paciente desarrolle la adherencia al tratamiento y busque el sentido positivo de la enfermedad (Torres, 2010).

Este programa ayudó a que los mecanismos de adaptación se ubicaran en el nivel compensatorio, en tanto que ayudan al individuo a buscar respuestas adaptativas que regulen su manera de actuar, favoreciendo así el afrontamiento de la enfermedad; se trabajó con las estrategias personales para que los participantes cuidaran su salud; en el ámbito afectivo, se aplicaron técnicas para el manejo de las emociones y la ansiedad; se utilizaron técnicas de relajación, autoestima y autorreconocimiento. Los mecanismos sociales tienen que ver con el rol dentro de la familia, las relaciones con amigos y compañeros de trabajo, de modo que al mejorar el manejo de emociones las relaciones sociales generaron un ambiente más cordial y menos hostil. A partir del programa se incrementaron los mecanismos sociales, mismos que no implicaban al comienzo una relación significativa, lo que es uno de los aportes de la presente investigación.

En conclusión, el programa de intervención psicológica mostró resultados positivos y permitió aumentar los mecanismos de adaptación de las personas con enfermedad renal crónica en estadio 4-5. En cuanto a la adherencia al tratamiento, el incremento no fue significativo; sin embargo, se obtuvo una mejor respuesta en el control de la ingesta de medicamentos y alimentos. Se sugiere en futuras investigaciones evaluar el tipo de actividades realizadas, su ejecución en tiempos precisos y las creencias de los participantes en los psicólogos en aras de lograr una adherencia al tratamiento más exitosa.

En el referido hospital, las personas con ERC no habían tenido un contacto con el área de Psicología, por lo que este programa logró ese acercamiento a través de la intervención y fomentó la idea de establecer un mejor trabajo multidisciplinario al subrayar los beneficios que dicho trabajo trae consigo, además de sensibilizar al personal de salud como un eslabón necesario para aumentar la adherencia al tratamiento y los mecanismos de adaptación en los participantes. 


\section{REFERENCIAS}

Córdoba, M.A. (2010). El abordaje psicológico desde Ellis en pacientes que padecen de insuficiencia renal crónica en edades comprendidas entre los 30 y 65 años del área de Hemodiálisis del Hospital Carlos Andrade Marín. Tesis inédita de Licenciatura. Quito: Universidad Politécnica Salesiana.

Devia, K., Falcón, A., Farfán, M. y Vargas, E. (2010). Mecanismos de adaptación de los pacientes con enfermedad renal crónica en terapia de hemodiálisis en la Unidad de Diálisis Lara II C.A. Barquisimeto. Revista de Medicina y Ciencias de la Salud, $11(3), 1-66$.

García, D., Ochoa, M., Martínez, N., González, B., Sánchez, M. y Martínez, M. (2016). Prevalencia de los mecanismos de adaptación del paciente con enfermedad renal bajo tratamiento de hemodiálisis. Cuidarte, 7(1), 1144-1151.

García, S., Bados, A. y Saldaña, C. (2012). Tratamiento cognitivo- conductual de una persona con trastorno de ansiedad generalizada. Anuario de Psicología, 42(2), 245-258.

Instituto Nacional de Estadística y Geografía (2018). Principales causas de mortalidad por residencia habitual, grupos de edad y sexo del fallecido. Consulta de resultados: Tabulados básicos. México: INEGI. Recuperado de https://www.inegi.org.mx/ sistemas/olap/registros/vitales/mortalidad/tabulados/ConsultaMortalidad.asp.

Martín A., L. y Grau A., J.A. (2014). La investigación de la adherencia terapéutica como un problema de la psicología de la salud. Psicología y Salud, 14(1), 89-99.

Martínez, C. y Grupo de Redactores (2014). Documento de consenso para la detección y manejo de la enfermedad renal crónica. Nefrología, 34(2), 243-262. Recuperado de http://scielo.isciii.es/pdf/nefrologia/v34n2/documento_consenso.pdf.

Mendoza M., H., González V., R., Cabrera F., N.L. y Arzate R., R. (2016). Impacto de la atención psicológica en el paciente con insuficiencia renal crónica. Revista Electrónica de Psicología Iztacala, 19(3), 972-993.

Molina, J., Sánchez, C., Castro, M. y Morera, S. (2014). Aspectos clínicos, preclínicos, terapéuticos y sociales de la insuficiencia renal crónica. Revista Electrónica de Veterinaria, 15(1), 1-17.

Oblitas, L. (2009). Psicología de la salud y calidad de vida (3a ed.). México: Cengage Learning.

Organización Mundial de la Salud (2004). Adherencia a los tratamientos a largo plazo: pruebas para la acción. Ginebra: OMS. Recuperado de http://www.paho.org/hq/index.php?option=com_docmanytask=doc_viewygid=18722yItemid.

Organización Mundial de la Salud (2016). Enfermedad crónica del riñón: Materiales de comunicación. Ginebra: OMS. Recuperado de http://www.paho.org/hq/index.php?option=com_topicsyview=readallycid=7682yItemid=40937ylang=es.

Organización Mundial de la Salud (2016). Salud mental: fortalecer nuestra respuesta. Ginebra: OMS. Recuperado de http://www. who.int/mediacentre/factsheets/fs220/es/.

Organización Mundial de la Salud (2017). Día mundial de la salud: La depresión encabeza la lista de causas de enfermedad. Ginebra: OMS. Recuperado de http://www.who.int/campaigns/world-health-day/2017/es/.

Organización Panamericana de la Salud (2015). La OPS/OMS y la Sociedad Latinoamericana de Nefrología llaman a prevenir la enfermedad renal y a mejorar el acceso al tratamiento. Washington, D.C. Recuperado de http://www.paho.org/hq/index.php?option $=$ com_contentyview $=$ articleyid=10542\%3A2015-opsoms-sociedad-latinoamericana-nefrologia-enfermedad-renal-mejorar-tratamientoyItemid=1926ylang=es.

Ortiz, A. y Riobó, S. (2012). Nutrición e insuficiencia renal crónica. Nutrición Hospitalaria, 5(1), 41-52.

Pascual, V., Serrano, A., Pedro B., J., Ascaso, J., Barrios, V., Millán, J. y Cases, A. (2017). Enfermedad renal crónica y dislipidemia. Clínica e Investigación en Arteriosclerosis, 29(1), 22-35.

Pedreira R., G., Vasco G., A., Martínez D., Y., Herrera M., C., Baz R., M. y Junyent I., E. (2020). Déficit de actividades recreativas en hemodiálisis. Satisfacción y coste económico ante un proyecto lúdico terapéutico. Enfermería Nefrológica, 23(1), 83-92.

Pelechano, V. (2012). Intervención psicológica en enfermos crónicos: sentido común y sabiduría popular. Análisis y Modificación de Conducta, 38(157/158), 1-26.

Ramírez, B. (2016). Logoterapia y tanatología. Tejiendo una gran red para la creación del hospice vida, esperanza y amor. Revista Mexicana de Logoterapia, 5, 64-90.

Rodríguez, M., Rentería, A. y García, J. (2013). Adherencia a la dieta en pacientes diabéticos: Efectos de una intervención. Summa Psicológica, 10(1), 91-101.

Soria, R., Vega, C. y Nava, C. (2009). Escala de adherencia terapéutica para pacientes con enfermedades crónicas, basada en comportamientos explícitos. Alternativas en Psicología, 14(20), 89-103.

Torres C., S. (2010). Diseño, implementación y evaluación de un programa de intervención educativa para pacientes con insuficiencia renal crónica. Revista Latinoamericana de Medicina Conductual, 1(1), 37-46. 